\title{
Second Fatal Case of Infective Endocarditis caused by Gemella bergeriae
}

\author{
Aijan Ukudeeva, $\mathrm{MD}^{1 *}$; Hal K. Hawkins, MD, $\mathrm{PhD}^{1,2}$; Masood Ahmad, MD²; \\ Peerawut Deeprasertkul, $\mathrm{MD}^{2}$; Liliana Rincon, $\mathrm{MD}^{2}$; Brandon K. Kimbrel, BS${ }^{2}$; \\ David H. Walker, $\mathrm{MD}^{2}$ \\ ${ }^{1}$ Shriners Burns Hospital for Children, Galveston, TX, USA \\ ${ }^{2}$ University of Texas Medical Branch, Galveston, TX, USA
}

\begin{abstract}
Our case illustrates a fatal course of infection with Gemella bergeriae endocarditis that was complicated by cardiogenic shock due to perforation of the mitral valve with severe mitral regurgitation, extension of infection into the myocardium adjacent to the mitral valve, and coronary sinus thrombosis. (Int J Biomed. 2017;7(1):63-66.)
\end{abstract}

Key Words: Gemella bergeriae • infective endocarditis • mitral valve • cardiogenic shock

\section{Abbreviations}

AO, aorta; AML, anterior mitral leaflet; IE, infective endocarditis; LA, left atrium; LV, left ventricle; MV, mitral valve; MR, mitral regurgitation; PML, posterior mitral leaflet; PCR, polymerase chain reaction; RV, right ventricle

\section{Introduction}

The incidence of infective endocarditis (IE) in developed countries has increased dramatically during the last few decades. ${ }^{[1-4]}$ Between 2000 and 2011, the incidence of IE in the United States increased from 11 per 100,000 people to 15 per 100,000 people. ${ }^{[1]}$ Gemella bergeriae (or bergeri), one of the six species belonging to the genus Gemella, was isolated for the first time by Collins et al. ${ }^{[5]}$ in 1998 from the blood cultures of six febrile patients, three of whom had endocarditis. Since then, only seven cases of G.bergeriae endocarditis have been reported. ${ }^{[5-8]}$ This report describes a fatal case of IE caused by G.bergeriae, which is part of the normal flora of the human oral cavity, slow growing fastidious bacteria, which can insidiously lead to a fulminant fatal outcome..$^{[5-7,9-14]}$ We present the second fatal case of IE caused by G.bergeriae.

*Corresponding author: Aijan Ukudeeva, MD, MS. Shriners Burns Hospital for Children, Galveston, TX, USA.E-mail: draijan@ yahoo.com

\section{Case Presentation}

A63-year-old man with a medical history of hypertension and poliomyelitis was admitted to the emergency department with acute bilateral lower extremity weakness and pain, low back pain, and a 2-week history of influenza-like symptoms. On examination, his temperature was $96.8^{\circ} \mathrm{F}$, blood pressure was $98 / 44 \mathrm{mmHg}$, heart rate was 112 beats/min, and respiratory rate - 26/min. Cardiac examination revealed holosystolic murmur at the apex with radiation to the axilla. A physical exam revealed the decreased movement and sensation in both lower extremities; they were cool to the touch and mottled; a Doppler posterior tibial pulse and a Doppler dorsalis pedis pulse were not present. Laboratory investigations revealed a white blood cell count of $31.4 \times 10^{3} / \mu \mathrm{L}$, lactic acid of 6.3 $\mathrm{mmol} / \mathrm{L}$, B-type natriuretic peptide of $>3000 \mathrm{pg} / \mathrm{mL}$, and troponin I of $1.710 \mathrm{ng} / \mathrm{mL}$. Computed tomography of the abdomen and pelvis showed almost the complete occlusion of the abdominal aorta distal to the inferior mesenteric artery and the right common iliac artery. On the same day, the patient underwent aortoiliac thrombectomy through bilateral femoral 
incisions. Transthoracic echocardiogram showed bileaflet prolapse with leaflet thickening and large mobile vegetation $(1.1 \times 1.4 \mathrm{~cm})$ on the posterior mitral leaflet (Figures 1 and 2). Blood cultures were collected before starting antimicrobial therapy. Starting therapy included once-daily doses of vancomycin $1500 \mathrm{mg}$, piperacillin-tazobactam $4500 \mathrm{mg}$, and levofloxacin $750 \mathrm{mg}$. On the next day, blood cultures yielded gram-positive cocci in clusters, and the patient was treated with cefazolin $1000 \mathrm{mg} \mathrm{q} 8 \mathrm{~h}$. On the third day of his hospital stay, piperacillin/tazobactam $3375 \mathrm{mg}$ q6h and vancomycin $1000 \mathrm{mg} \mathrm{q} 12 \mathrm{~h}$ were started, and cefazolin was discontinued. His condition declined, and he developed oliguria. On the fourth day, the patient underwent intra-aortic balloon pump placement for afterload reduction. Coronary angiography showed nonocclusive coronary artery disease. On the fifth day, he developed respiratory distress and lactic acidosis of $9.3 \mathrm{mmol} / \mathrm{L}$ and was intubated. A few hours after intubation, he developed hypotension and bradycardia; cardiopulmonary resuscitation was initiated but was not successful.

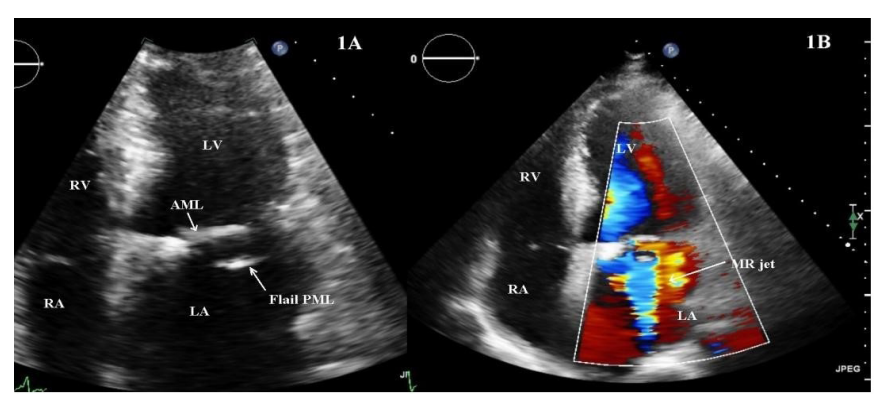

Fig. 1A. Apical four-chamber view showing a large vegetation and a portion of flail posterior leaflet (arrow).

Fig. 1B. Apical four-chamber view showing mitral regurgitation jet (arrow).

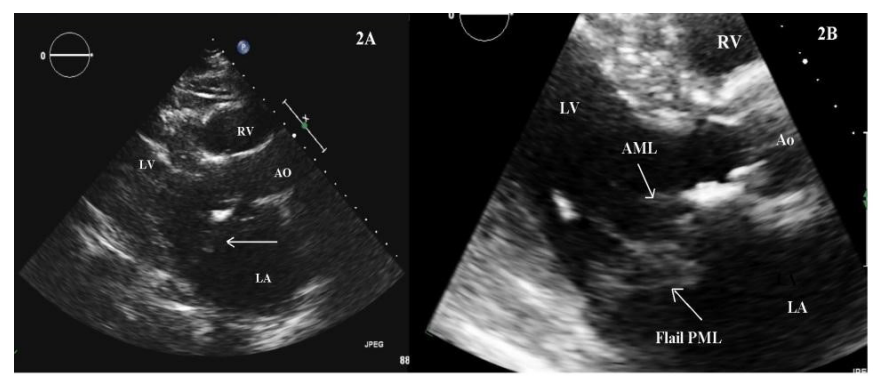

Fig. 2A. Parasternal long axis view, arrow pointing to mitral valve leaflets prior to endocarditis (arrow).

Fig. 2B. Parasternal long axis view, arrow pointing to a large vegetation, and flail portion of $P M L$.

Two days post-mortem, the final results of the first blood culture obtained in the emergency department became available, and the Gram-positive cocci were identified as Gemella bergeriae by MALDI-TOF mass spectrometry (bioMerieux, Durham, NC). However, the FDA has not approved this mass spectrometry for G.bergeriae investigation. Therefore, additional identification was performed by using the VITEK®2 (bioMerieux, Durham, NC), which also identified the organism as G.bergeriae. It was later determined that the patient had had an extensive dental procedure three weeks prior to his clinical presentation.

Post-mortem examination revealed massive cardiomegaly $(1100 \mathrm{~g})$, bilateral pulmonary congestion, and passive congestion of the liver. Both leaflets of the mitral valve were thickened. The posterior leaflet was perforated and had a $3 \times 2 \times 1 \mathrm{~cm}$ vegetation attached to the superior surface (Figures $3 \mathrm{~A}$ and $3 \mathrm{~B}$ ). The posterior-inferior wall of the left atrium and the posterior-superior wall of the left ventricle were inflamed. Inflammation involved the adjacent myocardium and pericardium. Adjacent to the inflamed myocardium, the coronary sinus contained a thrombus measuring $2 \times 1 \times 0.5 \mathrm{~cm}$. The upper lobe of the left lung had a small thromboembolus. Based on the autopsy findings and the clinical history, the cause of death was reported as cardiogenic shock due to perforation of the mitral valve with severe mitral regurgitation, extension of infection into the myocardium adjacent to the mitral valve, and coronary sinus thrombosis.

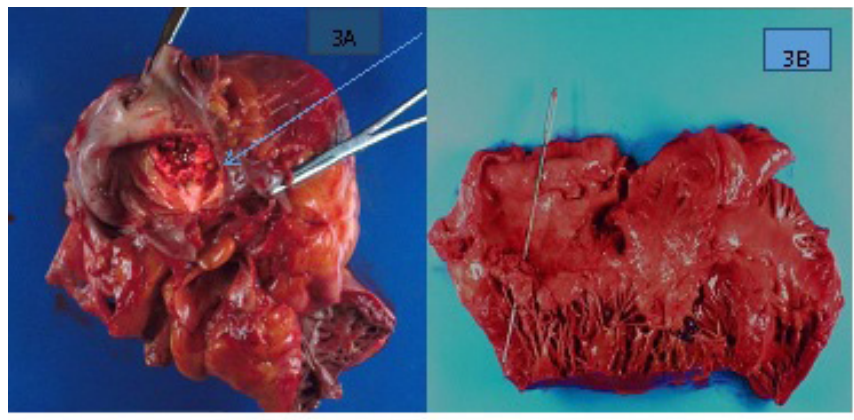

Fig. 3A. View from LA. Mitral valve endocarditis. Arrow pointing to the atrial aspect of $P M L$ with a large irregular reddish tan vegetation and perforation.

Fig. 3B. View of the opened left atrium and ventricle at autopsy. Mitral valve endocarditis. The probe passes through a hole in the vegetation that perforated $P M L$ The underlying chorda is thickened.

\section{Discussion}

The genus Gemella is comprised of catalase-negative, gram-positive cocci occurring in pairs (commonly with adjacent sides flattened), tetrads, and/or short chains. ${ }^{[15]}$ Six species, namely, G.haemolysans, G.morbillorum, G.bergeriae, G.sanguinis, G.palaticanis, and G.cuniculi, are currently recognized. ${ }^{[5,15-17]}$ The identification of Gemella isolates represents a challenge to clinical laboratories. Manual or commercial phenotypic methods may result in misidentification of Gemella spp. as viridans group streptococci or other related organisms and vice versa. ${ }^{[15,18]}$

$\mathrm{M}$ Collins and co-workers ${ }^{[5]}$ used 16S rRNA gene sequence analysis and an extensive repertoire of biochemical tests to characterize 6 strains of previously undescribed grampositive, facultatively anaerobic cocci recovered from blood cultures of hospitalized patients (half of whom were diagnosed with subacute bacterial endocarditis), leading to their proposal of a new Gemella species, G.bergeriae, to which these strains belonged. 
Pre-existing damaged heart valves, poor dental hygiene, intravenous drug abuse, and extensive dental procedures have been reported as the main predisposing risk factors for endocarditis due to G.bergeriae. ${ }^{[9,19]}$ To date, approximately 45 cases of endocarditis associated with Gemella spp. have been reported in the literature ${ }^{[5,7,8,12,20]}$ and among them only 8 were caused by G.bergeriae, including 7 adult cases and 1 pediatric case..$^{[5-7,9,10,13]}$ The majority of the patients described in the literature with G.bergeriae endocarditis had a good response to antibiotic therapy. ${ }^{[5-7,9,10,12,13]}$ In 2014 , Hussain et al. ${ }^{[7]}$ described the first fatal case of G.bergeriae infection, in which the patient with a history of rheumatic heart disease developed an embolic stroke as a complication of IE. Despite antibiotic therapy and intensive treatment, the patient expired due to intracerebral and subarachnoid hemorrhage secondary to rupture of a mycotic aneurysm in the right middle cerebral artery.

In 2007, Stroup et al. ${ }^{[11]}$ described another fatal case of IE in which bacteria of the genus Gemella were identified without species identification. That patient was diagnosed as having IE with Gram-positive cocci, and on day 2 developed embolic strokes. On day 8 , the patient had cardiogenic shock due to perforation of the anterior mitral leaflet and severe mitral regurgitation. This patient underwent valve replacement; on postoperative day 6 the patient expired due to intracranial hemorrhage from the right middle cerebral artery. Blood cultures of this patient on day 3 identified only Gram-positive cocci, and further study of the blood culture and tissue of the mitral valve identified the organism as the genus Gemella without determination of the species.

In 2015, Pachirat et al. ${ }^{[9]}$ described the first case of tricuspid valve IE in which G.bergeriae was identified. The organism was identified by PCR sequencing of DNA from tissues of the patient's cardiac valve despite negative cultures. The patient was discharged home with a good outcome.

In our case, the patient had a long history of mitral valve prolapse. He had an extensive dental procedure three weeks prior to his clinical presentation. He developed influenza-like symptoms 2 days prior to his admission to the emergency department with lower extremity weakness and pain due to a septic embolus to the abdominal aorta. On the fifth day after admission, the patient expired due to cardiogenic shock. The results of the patient's blood culture were later reported as

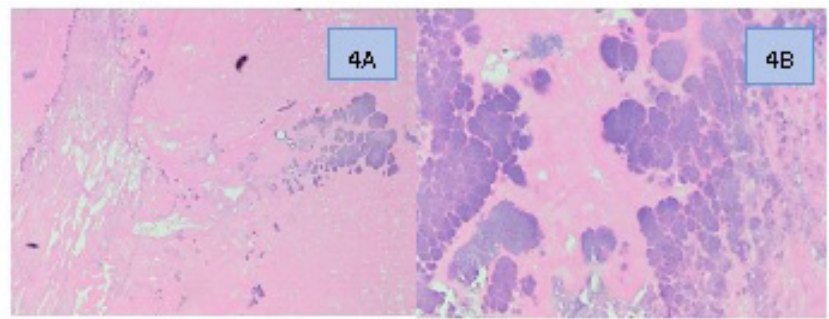

Fig. $4(\boldsymbol{A}, \boldsymbol{B}, \boldsymbol{C})$. Micrographs showing sections of the vegetation on $P M L, H \& E$ staining. Large blue-staining colonies of bacteria are present $(\boldsymbol{A}$ and $\boldsymbol{B})$. Necrosis of myocardium $(\boldsymbol{B})$ extending to coronary sinus, which contains a large thrombus $(\boldsymbol{C})$. positive for G.bergeriae. Postmortem histology (Fig.4) of the mitral valve and cardiac tissue revealed structures consistent with colonies of lysed bacteria.

Based on the cited literature, it is appropriate to question the general opinion that Gemella spp. are harmless microorganisms. ${ }^{[10]}$ G.bergeriae endocarditis can have a fulminant course with serious embolic complications..$^{[7]}$ Patients suspected to have Gemella endocarditis should be treated immediately with antibiotics due to the potentially aggressive character of this infectious process..$^{[7,10-12]}$ Empiric treatment for Gemella endocarditis with penicillin/ vancomycin and an aminoglycoside has been recommended ${ }^{[3]}$ to prevent a destructive course and avoid a fatal outcome. Prompt antibiotic treatment is the best way to decrease the risk of embolic complications. ${ }^{[3,7]}$

\section{Competing interests}

The authors declare that they have no competing interests.

\section{References}

1. Pant S, Patel NJ, Deshmukh A, Golwala H, Patel N, Badheka A, et al. Trends in infective endocarditis incidence, microbiology, and valve replacement in the United States from 2000 to 2011. J Am Coll Cardiol. 2015; 65(19):2070-6. doi:10.1016/j.jacc.2015.03.518

2. Griffin MR, Wilson WR, Edwards WD, O'Fallon WM, Kurland LT. Infective endocarditis. Olmsted County, Minnesota, 1950 through 1981. JAMA. 1985;254(9):1199-202.

3. Habib $\Gamma$, Hoen B, Tornos P, Thuny F, Prendergast B, Vilacosta I, et al.; ESC Committee for Practice Guidelines. Guidelines on the prevention, diagnosis, and treatment of infective endocarditis (new version 2009): the Task Force on the Prevention, Diagnosis, and Treatment of Infective Endocarditis of the European Society of Cardiology (ESC). Endorsed by the European Society of Clinical Microbiology and Infectious Diseases (ESCMID) and the International Society of Chemotherapy (ISC) for Infection and Cancer. Eur Heart J. 2009; 30(19):2369-413. doi: 10.1093/eurheartj/ ehp285.

4. Yew HS, Murdoch DR. Global trends in infective endocarditis epidemiology. Curr Infect Dis Rep. 2012;14(4):367-72.doi: 10.1007/s11908-012-0265-5.

5. Collins MD, Hutson RA, Falsen E, Sjöden B, Facklam RR. Gemella bergeriae sp. nov., isolated from human clinical specimens. J Clin Microbiol. 1998;36(5):1290-3.

6. Logan LK, Zheng X, Shulman S. Gemella bergeriae endocarditis in a boy. Pediatr Infect Dis J. 2008;27(2):184-6. doi: 10.1097/INF.0b013e31815b1af0.

7. Hussain K, Abubaker J,AlDeesi ZO,Ahmed R. Unreported neurological complications of Gemella bergeriae infective endocarditis. BMJ Case Rep. 2014; pii: bcr2014204405. doi: 10.1136/bcr-2014-204405.

8. Elsayed S, Zhang K. Gemella bergeriae endocarditis diagnosed by sequencing of rRNA genes in heart valve tissue. J Clin Microbiol. 2004;42(10):4897-900.

9. Pachirat O, Watt G, Pussadhamma B. First Case of Tricuspid Valve Endocarditis Caused by Gemella bergeri. Case Rep Med. 2015;2015:704785. doi: 10.1155/2015/704785.

10. Purcell LK, Finley JP, Cohen R, Lovgren M, Halperin 
SA. Gemella species endocarditis in a child. Can J Infect Dis. 2001;12(5):317-20.

11. Stroup JS. Bransteitter BA, Reust R. Infective endocarditis caused by Gemella species. Infect Dis Clin Pract. 2007;15(3):203-5.

12. Vasishtha S, Isenberg HD, Sood SK. Gemella morbillorum as a cause of septic shock. Clin Infect Dis. 1996; 22(6):1084-6. 13. Virgilio E, Chieco PA. Sixth case of infective endocarditis caused by Gemella bergeri. Braz J Infect Dis. 2014;18(4):467. doi: 10.1016/j.bjid.2014.05.002.

14. Zakir RM, Al-Dehneh A, Dabu L, Kapila R, Saric M. Mitral bioprosthetic valve endocarditis caused by an unusual microorganism, Gemella morbillorum, in an intravenous drug user. J Clin Microbiol. 2004; 42(10):4893-6.

15. Ruoff K. Aerococcus, Abiotrophia, and other infrequently isolated aerobic catalase-negative, grampositive cocci. In: Murray PR, Baron EJ, Jorgensen JH, Pfaller MA, Yolken RH, editors. Manual of clinical microbiology. 8th ed. American Society for Microbiology, Washington, D.C. 2003:434-444.

16. Collins MD, Rodriguez Jovita M, Foster G, Sjödén B, Falsen E. Characterization of a Gemella-like organism from the oral cavity of a dog: description of Gemella palaticanis sp. nov. Int J Syst Bacteriol. 1999;49 Pt 4:1523-6.

17. Hoyles L, Foster G, Falsen E, Collins MD. Characterization of a Gemella-like organism isolated from an abscess of a rabbit: description of Gemella cunicula sp. nov. Int J Syst Evol Microbiol. 2000;50 Pt 6:2037-41.

18. Woo PC, Lau SK, Fung AM, Chiu SK, Yung RW, Yuen KY. Gemella bacteremia characterized by $16 \mathrm{~S}$ ribosomal RNA gene sequencing. J Clin Microbiol. 2003;56(9):690-3. 19. Al-Hujailan G, Lagacé-Wiens P. Mechanical valve endocarditis caused by Gemella morbillorum. J Med Microbiol. 2007;56(Pt 12):1689-91.

20. Al Soub H, El-Shafie SS, Al-Khal AL, Salam AM. Gemella morbillorum endocarditis. Saudi Med J. 2003;24(10):1135-7. 